\title{
ESCUELAS INCLUSIVAS EN EL CONTEXTO DE SEGREGACIÓN SOCIAL DEL SISTEMA ESCOLAR CHILENO
}

\section{Iván Ortiz ${ }^{1}$}

\begin{abstract}
RESUMEN
Este estudio aporta nueva evidencia de los efectos composicionales de las escuelas sobre el aprendizaje de los estudiantes. Primero, averigua en qué medida existen establecimientos con mixtura social, a pesar de la segregación social del sistema escolar chileno. Los establecimientos inclusivos y segregados se comparan respecto de sus características generales y en cuanto a las oportunidades de aprender matemática ofrecidas a los estudiantes. Segundo, analiza el rendimiento en la prueba de matemática PISA 2012 de los estudiantes de los establecimientos segregados e inclusivos, considerando la diversidad social en su interior. Los resultados muestran que un 23\% de los alumnos de 15 años asiste a escuelas inclusivas. En ellas los estudiantes de niveles socioeconómicos inferiores obtienen mejores puntajes que lo predicho a partir de sus antecedentes socioculturales; lo contrario sucede a los estudiantes de niveles socioeconómicos superiores. Los establecimientos segregados e inclusivos difieren en cuanto a las oportunidades de aprender matemática ofrecidas a los estudiantes.
\end{abstract}

Palabras clave: segregación social, equidad de la educación, resultados de aprendizaje, efecto de los pares.

\section{INCLUSIVE SCHOOLS IN THE CONTEXT OF SOCIAL SEGREGATION IN THE CHILEAN SCHOOL SYSTEM}

\begin{abstract}
This study provides new evidence of the compositional effects of schools on student learning. First, it tries to find out to what extent, despite the social segregation of the Chilean school system, there are inclusive schools. Comparisons are made of inclusive and segregated schools on some general characteristics and for the mathematics learning opportunities offered to students. Second, the performance of students on the mathematics test PISA 2012 is analyzed; inclusive and segregated schools are compared, considering their social diversity. The results show that 23\% of 15 year olds attend inclusive schools. In these schools, students from lower socioeconomic levels score higher than predicted given their socio-cultural backgrounds; the opposite occurs with students from higher socioeconomic levels. The segregated and inclusive institutions differ in regards to the mathematics learning opportunities offered to students.
\end{abstract}

Keywords: social segregation, equity in education, learning outcomes, peer effect.

1 Facultad de Educación, Universidad Alberto Hurtado, Santiago, Chile. Contacto: ivanortizc@ gmail.com 


\section{Antecedentes}

La investigación educacional comparada ha dedicado muchas páginas a examinar la (in)equidad del aprendizaje, entre diversos grupos de estudiantes y de escuelas de distintos países. Los informes de resultados del Programme for International Student Assessment (PISA) y del Trends in International Mathematics and Science Study (TIMSS) son ejemplos paradigmáticos al respecto ${ }^{2}$. Estos estudios han mostrado insistentemente que el nivel socioeconómico (NSE) de las familias de los estudiantes y el de las escuelas a donde asisten está asociado al rendimiento académico; y que el efecto del NSE de la escuela es independiente y superior al efecto del NSE del estudiante a nivel individual (por ejemplo, OECD, 2013; Mullis y otros, 2012).

Existen sociedades donde la capacidad predictiva del NSE de los estudiantes sobre el rendimiento es más fuerte que en otras. De acuerdo con OECD (2013), la capacidad predictiva del NSE sobre el rendimiento en Chile es una de las más fuertes a nivel internacional: explica el 23,1\% de la varianza del rendimiento en matemática. Por otra parte, en algunos países, la desigualdad socioeconómica de los estudiantes, sea esta alta o baja, se manifiesta en cada escuela; en este caso se habla de escuelas inclusivas, heterogéneas, con mixtura social. En Chile, en cambio, las escuelas tienden a diferenciarse según el NSE, concentrando estudiantes de un NSE similar (escuelas segregadas, homogéneas). Estudios que cuantifican la segregación social del sistema escolar chileno sugieren que es muy alta en comparación con otros países (por ejemplo, Valenzuela, Bellei y De los Ríos, 2009; OECD, 2010; OECD, 2013).

¿Qué consecuencias tiene la mayor o menor segregación social del sistema escolar? La segregación social se acompaña de segregación

2 PISA es un estudio periódico realizado cada tres años por la Organización para la Cooperación y el Desarrollo Económicos OCDE (Organisation for Economic Co-operation and Development, OECD), que busca evaluar en qué medida los estudiantes de 15 años han adquirido competencias esenciales para una completa participación en la sociedad. Se evalúan las áreas de Lectura, Ciencias Naturales y Matemática. Se aplica en todos los países de la OCDE y en otros que deciden participar. TIMSS también es un estudio internacional periódico del rendimiento en Matemática y Ciencias de estudiantes de $4^{\circ}$ y $8^{\circ}$ grado; es organizado y liderado por la International Association of Assessment. 
académica, en el sentido de que la distribución del logro académico de las escuelas sigue un patrón similar a la de su NSE. En la evaluación PISA 2012, más del 75\% de las diferencias de rendimiento entre las escuelas chilenas es explicado por el NSE de la escuela (OECD, 2013). La segregación tiene efectos negativos para los resultados académicos de los estudiantes concentrados en escuelas de bajo NSE, y positivas para aquellos reunidos en escuelas de alto NSE, hecho ampliamente descrito por la literatura y confirmado también para el caso de Chile (MacEwan, 2003; Sapeli \& Vial, 2005; Valenzuela et al., 2009; OECD, 2010; OECD, 2013; Cerón y Lara, 2011; Mizala \& Torche, 2012).

\subsection{Efectos de la mixtura social en las escuelas}

La investigación internacional ha podido comprobar, además, que la mixtura social en las escuelas tiene efectos positivos para el aprendizaje de los estudiantes de NSE vulnerable (ver Harris 2010, para una revisión relativamente reciente). A nivel nacional, hay pocos estudios que pongan en evidencia este último punto. En su estudio sobre la segregación en el sector público y privado, Mizala y Torche (2012) dedican algunas líneas a señalar que la desviación estándar del NSE de las familias de los estudiantes al interior de las escuelas municipales (asociadas normalmente a un público escolar de escasos recursos) tiene una influencia positiva en los resultados del Sistema Nacional de Medición de la Calidad de la Educación (SIMCE), hecho que no se repite en las escuelas particulares subvencionadas; las autoras no analizan si el efecto positivo es parejo, o bien, se concentra en los estudiantes más o menos vulnerables de esas escuelas municipales. Más explícitos son los resultados encontrados por Taut y Escobar (2012), quienes reportan que la heterogeneidad social de las escuelas (no solo las municipales) tiene un efecto positivo en los resultados académicos medidos por el SIMCE, en el sentido de que los estudiantes de NSE bajo y medio obtienen mejores resultados en este tipo de escuelas que en aquellas de baja heterogeneidad social; se reportan diferencias sustantivas de 44 y 61 puntos en Lenguaje y Matemática, respectivamente, para los estudiantes de más bajo ingreso familiar.

En cambio, la evidencia es más bien negativa cuando se trata de determinar si el aprendizaje de alumnos socialmente favorecidos 
se ve también beneficiado en contextos escolares inclusivos. No hemos encontrado estudios que muestren evidencia de que también se benefician, en cambio existe literatura que pone en duda dicho beneficio. Una revisión de Bellei (2013) cita un estudio de Angrist $\&$ Lang del año 2004 como exponente de que tales contextos no alteran los resultados de estos estudiantes, y cita un estudio de Hoxby de 2000 y otro de Schindler del 2003 como exponentes de que los perjudican. Perry \& McConney (2010), con datos PISA de Australia, presentan evidencia de que este tipo de alumnos tiene logros más bajos cuando asisten a escuelas de menor NSE. A nivel nacional, el estudio de Taut y Escobar recién mencionado revela que los estudiantes de alto NSE se ven perjudicados en escuelas heterogéneas, pero por diferencias pequeñas de seis y ocho puntos menos de lo que ellos rinden en escuelas homogéneas, en Lenguaje y Matemática, respectivamente.

Las consecuencias de la segregación escolar no se limitarían al rendimiento de los estudiantes favorecidos y desfavorecidos en las pruebas de Lenguaje, Matemática o Ciencias. La segregación alteraría la calidad de la experiencia formativa de los alumnos en un sentido más amplio, al impedir que aprendan precozmente a convivir con respeto y tolerancia hacia los otros diferentes, y al impedir que la convivencia mutua genere principios de identificación social común, necesarios para los desafíos a nivel país de democracia y cohesión social (Peña, 2007). Si bien la escuela no es la única oportunidad que tienen los ciudadanos de hacer una experiencia formativa de inclusión, es razonable pensar que mientras más precozmente la hagan, más sólidos serían los efectos ${ }^{3}$. Se trata de expectativas razonables, pero necesitan apoyo empírico. Si la investigación local de las consecuencias de la segregación para el aprendizaje convencional es parcial, en este otro dominio de la formación, es inexistente.

3 En cualquier caso, no es razonable suponer que tales experiencias formativas vayan a ser logradas por el mero hecho de la mixtura social de las escuelas, en ausencia de proyectos educativos que procuren deliberadamente que ello ocurra. Manzi (2007) comenta varios estudios norteamericanos acerca del efecto del contacto entre estudiantes provenientes de diverso origen social o étnico sobre el prejuicio mutuo en la sala de clases; ellos muestran que cuando la interacción entre estudiantes de diverso origen se da en un contexto controlado que favorece el contacto interpersonal estrecho en un marco de cooperación, igualdad de trato y apoyo institucional, se obtiene un claro efecto positivo. 


\section{2. ¿Influencia de los pares?}

La literatura comparada ha hecho visibles -y deseables- sistemas educacionales socialmente inclusivos (por ejemplo, países escandinavos, Canadá y otros) donde las varianzas del NSE de los estudiantes y de sus puntajes académicos (altos, en general) se concentran al interior de las escuelas, siendo estas bastante similares entre sí. Tales sociedades exhiben también atributos de inclusión social en otros dominios de la vida colectiva, además de la escolar, lo que las hace más interesantes aún. Pero no es sencillo discernir cómo se relacionan esos distintos dominios y cuál dominio de inclusión social es precondición para otros.

La elucidación de los mecanismos y procesos a través de los cuales la segregación escolar produce las consecuencias en los aprendizajes descritas más arriba ha sido un tema de mucho interés teórico, metodológico y político, aunque no ha sido resuelto hasta ahora. El debate radica en si el mecanismo o proceso puede o no ser considerado un efecto de los pares (peer effect). En OECD (2012) se plantea que algunos de los efectos composicionales de las escuelas segregadas sobre el aprendizaje pueden ser atribuidos a la interacción entre pares. Por ejemplo, en escuelas socialmente aventajadas, el intercambio entre estudiantes talentosos puede ser formativo para ellos en la medida en que aprenden unos de otros. Sin embargo, este tipo de escuelas suele ofrecer a sus estudiantes un mejor ambiente de aprendizaje y recursos de enseñanza y, por esta vía, facilita su logro académico. Por su parte, Willms (2010) presenta evidencia de que casi la mitad de los efectos composicionales hallados en su análisis secundario de datos PISA 2006, corresponde a efectos de variables de la escuela, tales como la calidad de la instrucción, la cobertura curricular, la cantidad de horas de la asignatura en el plan de estudios y otras. En tanto Wilkinson (2002) distingue entre efectos composicionales y efecto de los pares, los cuales no deben considerarse sinónimos. Los primeros son definidos como efectos asociados a la agregación de características individuales de un grupo de estudiantes sobre sus resultados de aprendizaje, descontado el efecto de esas características consideradas a nivel individual; efectos que podrían ser atribuidos a diversos factores, entre ellos el clima disciplinario que 
los estudiantes en conjunto producen, o las prácticas docentes que se adaptan al colectivo. En cambio, un efecto de pares verdadero sería un caso especial de efectos composicionales: aquel que deriva de la influencia directa de los pares a través de dinámicas de interacción ${ }^{4}$.

En relación con la distinción anterior, Wilkinson et al. (2002) desarrollaron un modelo conceptual donde los efectos composicionales operan a través de una serie de niveles jerárquicos, desde las influencias del nivel de la escuela, a las del nivel de curso, luego a las influencias grupales intracurso, para llegar a las influencias de un ambiente configurado para el aprendizaje entre pares. Es interesante notar que los autores reconocen, por una parte, la reciprocidad de las influencias entre estudiantes, profesores y la organización y gestión de la escuela, lo que vuelve aún más complejo el discernimiento de efectos y por otra, asignan una influencia mayor a aquellos niveles donde los procesos de enseñanza y aprendizaje están ocurriendo. La implementación del currículo y los recursos de enseñanza jugarían un rol crucial, pero añaden que la optimización de la interacción entre métodos de enseñanza y oportunidades curriculares ocurriría en el nivel, no del curso como un todo, sino de sus grupos. En este lugar, los autores sugieren que se trataría de configurar interacciones entre pares que alteren el currículo y los métodos de enseñanza, de tal modo de ofrecer experiencias cualitativamente diferentes a los estudiantes, conforme a la composición del curso ${ }^{5}$. Así, la interacción entre pares no es para ellos una variable más en el estudio de los factores asociados al aprendizaje, sino una muy crucial y debe ser impulsada desde un punto de vista pedagógico.

Parr \& Townsend (2002) describen una serie de ambientes, procesos y mecanismos obrando en el verdadero efecto pares: ambientes de convivencia entre pares, normalmente autoseleccionados, donde existen normas o creencias compartidas favorables (o desfavorables) para el aprendizaje; por ejemplo, la creencia de que leer es positivo,

4 Bellei (2013) prefiere referirse a esta distinción como efectos de los compañeros, indirectos o directos.

5 Esta es una visión muy alineada con los partidarios de una pedagogía para aulas diversas, que se hace cargo de las diferencias en los niveles de aprendizaje que presentan los estudiantes al interior de cada aula (Tomlinson, 2005; Sousa \& Tomlinson, 2011). 
creencia que tal vez se generó por modelaje de algunos líderes del grupo. Otras fuentes para el efecto pares son aquellos ambientes configurados deliberadamente para generar interacciones cognitivas entre pares, en las cuales construyen juntos conocimiento, tales como tutorías, aprendizaje grupal cooperativo y colaborativo. En este tipo de espacios, los procesos psicosociales en juego no son tanto la imitación de modelos o el contagio de creencias, sino el conflicto cognitivo y la restructuración cognitiva. Sin embargo, Hattie (2002) está en desacuerdo con lo anterior. Su revisión de la literatura lo lleva a concluir que rara vez se atestigua el efecto directo de los pares y que su influencia, en el mejor de los casos, es indirecta, ya que a menudo lo que sucede con diversas composiciones de los cursos es que afectan la probabilidad de que reciban una enseñanza distinta. Cualquiera de las composiciones estudiadas por el autor (por grupos de habilidad, sexo, edad y tamaño de la clase) tiene menos consecuencias sobre el aprendizaje que la naturaleza y calidad de la instrucción en el aula conducida por los profesores.

Thrupp, Lauder y Robinson (2002) sostienen que después de décadas de investigación acerca de los efectos de composición o mixtura social de las escuelas, el consenso acerca de la naturaleza y tamaño de estos efectos es notablemente poco, una conclusión que Harris (2010) vuelve a confirmar años después. Según los primeros autores esto se explicaría, por una parte, por una inadecuada teorización y operacionalización del concepto y, por otra, por las dificultades inherentes a la medición de las variables que estarían implicadas. Desde un punto de vista metodológico, se comprende la dificultad inherente a la verificación del efecto directo de los pares en estudios correlacionales con datos de los sistemas escolares reales. La medición de variables de interacción entre pares y su inclusión en las ecuaciones de regresión, es dificultosa; por otro lado, no ocurre una asignación al azar de estudiantes a las escuelas, de modo que los sesgos de selección son prácticamente inevitables. Angrist (2014) sostiene, con base en meticulosos análisis econométricos, que los datos de los individuos y las características de los grupos a los que pertenecen están correlacionados poderosamente gracias a fuerzas mecánicas y estadísticas. Entonces, la correlación entre los resultados de un individuo y los del grupo al que pertenece (curso, escuela), 
aunque se controle por otras variables, va a resultar positiva, aún en casos en que conceptualmente no tiene sentido atribuir una relación causal. Por eso, el autor dice que hay que poner una vara muy alta para verificar verdaderos efectos de los pares. Una condición para ello es que los diseños de investigación deben distinguir entre quienes serían los sujetos de un efecto par y los pares que potencialmente proveerían el mecanismo para un efecto causal sobre esos sujetos. Estos diseños serían experimentales.

La evidencia empírica de efectos composicionales (a secas) no es difícil de generar; en cambio, la evidencia de los efectos directos de los pares, hasta ahora, suele descansar en la inferencia y la interpretación teóricas.

Pero hay excepciones. Carrell, Sacerdote $\&$ West (2013) llevaron a cabo un ingenioso estudio experimental de los efectos composicionales sobre el aprendizaje. En un primer momento asignaron al azar a una cohorte de jóvenes recién ingresados a la fuerza aérea norteamericana a diferentes escuadrones de unos 30 integrantes, los cuales seguían cursos y programas de estudios iguales, sin posibilidad de elección de los docentes. Los jóvenes fueron previamente evaluados en una serie de parámetros de rendimiento académico. Al finalizar el período de instrucción se halló que los jóvenes inicialmente evaluados con baja habilidad, se beneficiaron significativamente de haber estado con pares de mayor habilidad. Con base en los resultados de esta etapa, los investigadores sometieron a una nueva cohorte de cadetes al siguiente procedimiento, luego de ser evaluados en sus habilidades académicas. La mitad de ellos fue asignada al azar a sus escuadrones, mientras la otra mitad fue asignada conforme a un diseño para optimizar las posibilidades de logro de los jóvenes de más baja habilidad; estos fueron puestos en escuadrones solo junto con jóvenes de muy alta habilidad (escuadrones bimodales). Mientras que los jóvenes de habilidad intermedia fueron puestos en otros escuadrones (homogéneos). Contrariamente a lo esperado, los jóvenes de baja habilidad experimentaron un efecto negativo, con logros significativamente más bajos que en el grupo control, en cambio los estudiantes de alta habilidad no fueron afectados por el tratamiento. Por otra parte, los de habilidad intermedia también tuvieron un efecto 
positivo, cuando se había predicho que no serían afectados. Los autores explican, con base en los reportes de los jóvenes después del experimento, que los estudiantes de los escuadrones bimodales se segregaron en redes separadas según su habilidad. En comparación con el grupo control, los cadetes de baja habilidad tendieron a formar grupos pequeños de estudio con otros de baja habilidad. En cuanto a los estudiantes de habilidad intermedia, la evidencia sugiere que su efecto positivo se debe a que no interactuaron con jóvenes de baja habilidad. Así, el estudio muestra que la interacción entre pares sí juega un rol significativo en los procesos educativos.

El debate acerca de la importancia de los efectos composicionales está abierto a consideraciones políticas e ideológicas. Que el aprendizaje sea o no afectado por efectos composicionales atribuibles a factores de la escuela y/o a efectos de los pares tendría implicaciones diferentes para los esfuerzos de mejora educativa, según algunos (Thrupp et al., 2002; y Mizala \& Torche, 2012). Pues, si los efectos directos de los pares no juegan un rol importante, se podría pensar que la mejora del aprendizaje descansaría enteramente en variables que influencian la efectividad de la escuela en la provisión de la enseñanza y los recursos de aprendizaje. Por el contrario, si su rol es importante, las perspectivas de los esfuerzos y políticas de mejora se complejizan, pues la responsabilidad de las escuelas se vería limitada por reglas del juego de nivel macro, generadoras de segregación escolar. En este artículo se sugiere que la responsabilidad de la escuela está limitada por esas reglas del juego aún en ausencia del efecto directo de los pares, pues la segregación social también influye en el desempeño de la escuela como organización, en la factibilidad de entregar una enseñanza de calidad, por la vía de factores como las expectativas y la preparación de los docentes, la cobertura curricular y otros. Curiosamente, esta perspectiva de los efectos de la segregación no ha sido un tema de interés en la investigación.

\subsection{Propósitos del estudio}

Este estudio se propone aportar evidencia empírica de los efectos composicionales de las escuelas sobre el aprendizaje de los estudiantes. El debate técnico y político acerca de la segregación escolar en Chile 
ha puesto el énfasis en su radicalidad. En este contexto de alta segregación, ¿existen espacios inclusivos? No se encontraron estudios que pongan el foco de atención en la inclusión. En la medida en que los espacios actuales de mixtura social actual prefiguran lo que el país puede esperar de las políticas educacionales antisegregación, sería interesante conocerlos mejor.

Una vez identificados los establecimientos inclusivos se analizará el rendimiento académico diferencial de los estudiantes de distintos niveles socioeconómicos que los componen, para determinar si la mixtura social es o no un ambiente favorable para todos; los establecimientos inclusivos serán analizados en comparación con los establecimientos segregados. Respecto de este propósito, la evidencia chilena es insuficiente. Adicionalmente, los establecimientos inclusivos y segregados serán descritos en términos de algunas de sus características generales (como la dependencia administrativa). Interesa también describirlos en términos de algunas variables que pudieran estar relacionadas con el rendimiento en la prueba, como las oportunidades de aprender matemática que tuvieron los estudiantes. PISA incluyó por primera vez en la evaluación internacional 2012, una medición de qué y cuánta matemática se enseñó a los estudiantes, medición que el estudio internacional TIMSS venía haciendo desde sus comienzos. En efecto, el concepto de oportunidad de aprender fue desarrollado en el marco de las investigaciones de resultados de aprendizaje organizadas por la International Association of Assessment, para analizar las relaciones entre el currículo oficial, el currículo efectivamente implementado por las escuelas y el currículo aprendido por los alumnos (McDonnell, 1995). Específicamente, es entendido como el grado en que se ha enseñado a los alumnos los conocimientos contemplados en las pruebas administradas; incluye los eventos y actividades en los que han participado y que les dan la posibilidad de aprender aquello que se pregunta en estas pruebas (Schmidt, McKnight, Valverde, Houang \&Wiley, 1997). La inclusión de este concepto hace posible realizar una mejor interpretación de los datos, en tanto permite evitar interpretaciones simplistas de los resultados obtenidos por alumnos que han sido expuestos a distintas oportunidades de aprender. A su vez, puede entregar importantes señales a los sistemas educativos nacionales en términos de la 
distancia entre las prescripciones curriculares oficiales y la oferta curricular efectivamente implementada (Schmidt \& McKnight, 1995). El concepto se ha revelado como un buen predictor del rendimiento (Schmidt, Zoido \& Cogan, 2013) y un mediador entre diversas variables de efectividad, recursos y gestión de la escuela, y el rendimiento, en el entendido de que tales variables cobran sentido en la medida en que influyen en la calidad de la enseñanza en el aula.

Lamentablemente, las restricciones metodológicas de los datos impiden aportar evidencia que pueda ser interpretable como efecto de los pares, como hubiese sido deseable.

\section{Método}

El estudio se basa en un análisis secundario de los datos de Chile del estudio PISA 2012, con foco en el rendimiento en matemática. La muestra chilena se compone de 6.856 estudiantes de 15 años distribuidos en 221 establecimientos educacionales de todo el país. Se trata de una muestra extraída en dos etapas: establecimientos educacionales elegidos al azar, con una probabilidad proporcional a su tamaño, de acuerdo a los estratos definidos por el estudio; y luego estudiantes de 15 años elegidos al azar de cada establecimiento. El muestreo fue diseñado para optimizar la muestra de estudiantes de 15 años, más que para asegurar una muestra óptima de establecimientos educacionales.

El estudio siguió tres etapas. En la primera, la muestra de estudiantes fue dividida según el tipo de composición socioeconómica predominante en sus establecimientos: escuelas que agrupan preferentemente a estudiantes socialmente desaventajados, a estudiantes socialmente aventajados, o bien, a estudiantes de diversas condiciones socioeconómicas (o de una condición intermedia). Se usó el índice PISA de estatus económico, social y cultural por estudiante, el cual integra información del nivel de escolaridad de los padres, su nivel ocupacional y los bienes presentes en el hogar.

Para definir estos tres grupos, se calculó el promedio del índice PISA de estatus económico, social y cultural de cada escuela 
de la muestra y su diferencia con el promedio nacional. Con apoyo en la prueba t de Student, los establecimientos con promedios significativamente más bajos o más altos que el nacional se consideraron segregados desaventajados o aventajados, respectivamente, en tanto que aquellos cuyos promedios no eran significativamente distintos del nacional (estaban en el intervalo $+-1,96$ ) se consideraron mixtos o inclusivos. Este método de clasificación es reportado en OECD (2009) y usado en el informe de resultados PISA 2012 como un indicador de equidad (OECD, 2013).

Las escuelas cuyo NSE promedio no difiere significativamente del promedio nacional, pueden componerse, teóricamente, de estudiantes muy cercanos al NSE promedio nacional y/o de estudiantes de diferentes NSE, de tal modo que el promedio de ellos se acerque al promedio nacional. Luego de identificadas las escuelas de los tres grupos, se revisó la composición social interna de cada una, en términos de cuáles y cuántos quintiles de NSE de los estudiantes estaban presentes, para validar la clasificación anterior. Para la determinación de los quintiles nacionales de NSE se calcularon los percentiles 20, 40, 60, y 80 del índice PISA de estatus económico social y cultural, integrando los pesos de los estudiantes. La revisión verificó que el grupo mixto se componía de estudiantes de, al menos, cuatro quintiles de NSE. El cuadro siguiente muestra ejemplos de la composición social hallada en los establecimientos de los tres grupos. 
Cuadro 1: Ejemplos de la composición socioeconómica del alumnado en escuelas segregadas y mixtas. Cantidad de alumnos de cada quintil de NSE (Frecuencias brutas)

\begin{tabular}{|c|c|c|c|c|c|c|c|}
\hline $\begin{array}{c}\text { Tipo de composición } \\
\text { social }\end{array}$ & $N^{0}$ escuela & Quintil 1 & Quintil 2 & Quintil 3 & Quintil 4 & Quintil 5 & $\begin{array}{c}\text { Total } \\
\text { alumnos }\end{array}$ \\
\hline \multirow{10}{*}{$\begin{array}{c}\text { Segregadas } \\
\text { desaventajadas }\end{array}$} & 0000047 & 11 & 9 & 2 & 0 & 1 & 23 \\
\hline & 0000048 & 10 & 10 & 12 & 5 & 2 & 39 \\
\hline & 0000051 & 15 & 10 & 7 & 1 & 1 & 34 \\
\hline & 0000052 & 21 & 9 & 2 & 3 & 0 & 35 \\
\hline & 0000054 & 22 & 9 & 3 & 2 & 1 & 37 \\
\hline & 0000060 & 26 & 8 & 3 & 0 & 0 & 37 \\
\hline & 0000063 & 6 & 11 & 10 & 8 & 0 & 35 \\
\hline & 0000064 & 14 & 10 & 8 & 3 & 2 & 37 \\
\hline & 0000066 & 13 & 11 & 8 & 1 & 0 & 33 \\
\hline & 0000070 & 18 & 10 & 5 & 4 & 1 & 38 \\
\hline \multirow{10}{*}{ Mixtas } & 0000049 & 6 & 11 & 7 & 8 & 4 & 36 \\
\hline & 0000062 & 2 & 11 & 12 & 11 & 2 & 38 \\
\hline & 0000071 & 9 & 6 & 7 & 7 & 5 & 34 \\
\hline & 0000084 & 6 & 9 & 8 & 10 & 3 & 36 \\
\hline & 0000090 & 4 & 9 & 14 & 5 & 3 & 35 \\
\hline & 0000093 & 2 & 0 & 3 & 4 & 2 & 11 \\
\hline & 0000097 & 10 & 10 & 3 & 7 & 6 & 36 \\
\hline & 0000098 & 0 & 13 & 10 & 7 & 7 & 37 \\
\hline & 0000108 & 5 & 8 & 11 & 12 & 2 & 38 \\
\hline & 0000118 & 3 & 10 & 15 & 8 & 1 & 37 \\
\hline \multirow{10}{*}{$\begin{array}{l}\text { Segregadas } \\
\text { aventajadas }\end{array}$} & 0000036 & 0 & 0 & 2 & 9 & 25 & 36 \\
\hline & 0000037 & 1 & 6 & 8 & 8 & 8 & 31 \\
\hline & 0000038 & 0 & 0 & 3 & 16 & 21 & 40 \\
\hline & 0000040 & 0 & 0 & 0 & 3 & 35 & 38 \\
\hline & 0000041 & 0 & 0 & 2 & 6 & 26 & 34 \\
\hline & 0000042 & 0 & 2 & 6 & 16 & 15 & 39 \\
\hline & 0000043 & 0 & 0 & 0 & 4 & 34 & 38 \\
\hline & 0000044 & 0 & 0 & 1 & 2 & 22 & 25 \\
\hline & 0000046 & 0 & 0 & 0 & 1 & 36 & 37 \\
\hline & 0000050 & 0 & 0 & 0 & 3 & 34 & 37 \\
\hline
\end{tabular}

Fuente: bases de datos PISA 2012, elaboración propia. 
Posteriormente, se procedió a describir y comparar estos tres grupos en función de algunas variables de caracterización general de las escuelas, recogidas en el cuestionario para los directores ${ }^{6}$ :

- Dependencia administrativa del establecimiento. Se refiere a la pertenencia de los estudiantes a escuelas municipales, particulares subvencionadas, o particulares privadas.

- Tamaño de la localidad. Se refiere al tamaño en términos de la cantidad de habitantes de la localidad donde están emplazados los establecimientos de la muestra. Es una variable categorial.

- Selectividad académica. Corresponde a un índice simple con tres categorías de respuesta, elaborado a partir de dos ítems del cuestionario contestado por los directores: grado en que la escuela selecciona el ingreso de estudiantes por antecedentes académicos y grado en que el rendimiento académico constituye causal de transferencia de estudiantes a otro establecimiento.

La segunda etapa consistió en analizar el comportamiento en la prueba de matemática de los estudiantes de cada quintil de NSE, en los diferentes grupos de composición social. En particular, se realizó un análisis de los efectos de composición en cada grupo, mediante regresión lineal y análisis de residuos. Uno de los usos de la regresión lineal consiste en analizar los residuos de acuerdo a un criterio dado, el tipo de composición social de los establecimientos en este caso. Así, mediante regresiones lineales se predijo el rendimiento de los estudiantes a partir del índice NSE y se conservaron los residuos de cada caso. Luego, se calcularon los promedios de los residuos para cada quintil de NSE al interior de los tres grupos en estudio. Es decir, se obtuvo la diferencia entre el puntaje de matemática obtenido y el predicho por la regresión, para cada uno de los subgrupos recién mencionados.

Cuando los estudiantes de una muestra están anidados en escuelas, la literatura sugiere normalmente usar regresiones multinivel, lo que no fue necesario aquí. El anidamiento en escuelas tiende a homogeneizar a los estudiantes, violando el supuesto de

6 Dadas las características de la muestra, las variables de nivel escuela se deben analizar y reportar como atributos de los estudiantes. 
independencia de las observaciones. Pero la violación de tal supuesto no sesga los coeficientes de regresión, sino que subestima sus errores estándar (OECD, 2009), lo cual en este estudio es neutralizado gracias al tratamiento dado a la estimación del error estándar. En efecto, los procedimientos estadísticos establecidos por PISA prescriben que el cálculo del error estándar de cualquier parámetro poblacional debe ser replicado 80 veces mediante el método balanced repeated replication, para evitar el sesgo de subestimación.

Finalmente, se procedió a describir y a comparar estos tres grupos en función de algunas variables de efectividad escolar relacionadas con la calidad de la provisión de enseñanza ${ }^{7}$ :

- Oportunidad de aprender. Bajo este título se encuentran tres índices escalares construidos por PISA, a partir de varias preguntas hechas a los estudiantes respecto de las oportunidades que tuvieron de trabajar en aula ciertos tipos de contenidos y problemas de matemática: oportunidades de realizar tareas de matemática aplicada; oportunidades de realizar tareas de matemática pura o teórica; y oportunidades de oír diversos conceptos de matemática.

- Proporción de docentes certificados. Bajo este título se encuentran tres índices simples construidos a partir de preguntas hechas a los directores: la proporción de docentes titulados sobre el total de docentes de la escuela; la proporción de docentes con un grado de licenciatura, sobre el total de docentes de la escuela; y la proporción de docentes de matemática con un grado de licenciatura, sobre el total de docentes de matemática.

- Todos los análisis se realizaron conforme a los procedimientos estadísticos establecidos por PISA en OECD (2009). Aparte del cálculo riguroso del error estándar mencionado más arriba, también el rendimiento de los estudiantes en las pruebas de aprendizaje es calculado de manera especial. En vez de calcular la habilidad de los estudiantes mediante un puntaje único, se estima una distribución de probabilidades para cada uno de ellos, es decir, un rango de habilidad, del cual PISA entrega cinco valores plausibles escogidos al azar. Así, cualquier análisis relacionado

7 Son índices elaborados por PISA; la mayoría de los cuales son estandarizados, con el promedio de la muestra internacional fijado en 0 . 
con el rendimiento debe desarrollarse cinco veces, siendo su promedio el resultado final. Otra precaución en los análisis es la consideración de los pesos que deben ser asociados a cada estudiante y a cada escuela.

\section{Resultados}

A continuación se presentan los resultados obtenidos por el presente estudio.

\subsection{Composición social de los establecimientos}

El 42,8\% de los estudiantes de 15 años se encuentra en establecimientos segregados desaventajados; el 34,4\% se encuentra estudiando en establecimientos segregados aventajados; y el 22,8\% lo hace en establecimientos con mixtura social. Los valores del error estándar son 3,03; 3,45; y 2,44 respectivamente. Las escuelas del primer grupo alcanzan un promedio de 387,3 puntos en la prueba de matemática, en tanto que las escuelas inclusivas logran 407,2 puntos y las segregadas aventajadas, 477,7 puntos. El promedio del país fue de 423 puntos.

No obstante, al interior de cada uno de estos tres grupos de establecimientos, se encuentran estudiantes de los cinco quintiles de NSE en proporciones diversas de acuerdo al tipo de inclusión/ exclusión social que le es característico (ver Cuadro 2). En los segregados desaventajados, los quintiles 1 y 2 son mayoritarios, en tanto que los quintiles 3 y 4 son muy minoritarios; lo contrario sucede en el grupo de los segregados aventajados. El grupo de los establecimientos con mixtura social tiene una distribución semejante a la normal, con una presencia predominante del quintil 3. 
Cuadro 2: Distribución del alumnado por quintiles de NSE en los tres grupos de establecimiento. (Porcentajes de estudiantes y error standard)

\begin{tabular}{c|c|c|c|c|c|c}
\hline \multirow{2}{*}{ Quintil NSE } & \multicolumn{2}{|c|}{ Segregadas desaventajadas } & \multicolumn{2}{|c|}{ Mixtas } & \multicolumn{2}{c}{ Segregadas aventajadas } \\
\cline { 2 - 7 } & Porcentaje & Error st & Porcentaje2 & Error st2 & Porcentaje 3 & Error st3 \\
\hline Q1 & 38,4 & 2,26 & 13,7 & 1,21 & 1,7 & 0,39 \\
\hline Q2 & 28,4 & 1,04 & 24,9 & 1,79 & 6,4 & 0,79 \\
\hline Q3 & 19,3 & 1,27 & 27,4 & 1,56 & 15,6 & 1,21 \\
\hline Q4 & 11,3 & 0,89 & 23,8 & 1,22 & 28,9 & 1,22 \\
\hline Q5 & 2,6 & 0,37 & 10,3 & 0,97 & 47,4 & 2,52 \\
\hline Total & 100 & & 100 & & 100 & \\
\hline
\end{tabular}

Fuente: bases de datos PISA 2012, elaboración propia.

La revisión de la distribución de los quintiles en cada escuela, reveló que casi todas las escuelas segregadas desaventajadas tienen estudiantes en, al menos, cuatro quintiles; las escuelas compuestas solo de estudiantes de los quintiles 1 y 2, son casos raros. Esta situación contrasta con la distribución observada en las escuelas segregadas aventajadas, donde se encontraron muchas escuelas compuestas solo de compañeros(as) de los quintiles 4 y 5 , siendo predominante este último quintil. Este hallazgo motivó un análisis más detallado del grupo de escuelas segregadas aventajadas, el que reveló que las escuelas más segregadas en su interior corresponden a las particulares privadas. En este subgrupo, ningún estudiante pertenece al quintil $1 ;$ el $0,4 \%$ es del quintil 2; el 1,7\% es del quintil 3; apenas un 10,1\% es del quintil 4; y el $87,7 \%$ restante es del quintil 5 . Por contraste, una vez descontadas las escuelas de este subgrupo, las escuelas segregadas aventajadas que no son particulares privadas, quedan con una distribución interna menos segregada que el grupo original: $2,1 \% ; 8,1 \% ; 19,6 \% ; 34,6 \%$ y $35,6 \%$, para los quintiles 1 a 5 , respectivamente. Volveremos más adelante sobre este punto.

\subsection{Breve caracterización de los establecimientos segregados e inclusivos}

\subsubsection{Dependencia administrativa}

El Cuadro 3 muestra que la distribución de los estudiantes en establecimientos de los tres tipos de dependencia administrativa difiere en los tres grupos. Los estudiantes de las escuelas segregadas 
desaventajadas se distribuyen entre la dependencia municipal y la particular subvencionada, con ligero predominio de la primera. Los estudiantes de las escuelas mixtas también se distribuyen en estas dos dependencias, pero con cierto predominio de la segunda. Estos dos grupos de composición social no tienen presencia en la dependencia particular privada. Esto implica que todos los estudiantes particulares privados asisten a escuelas segregadas aventajadas. Sin embargo, no todos los estudiantes segregados aventajados son particulares privados; de hecho, la mayoría de ellos asiste a escuelas particulares subvencionadas e incluso una pequeña minoría va a establecimientos municipales. Según estos datos, la dependencia particular subvencionada presenta una situación más heterogénea que las otras, pues integra escuelas de los tres tipos de composición social de una manera relativamente balanceada. Al calcular la presencia de los tres grupos al interior de esta dependencia, se obtiene que 34\% de sus estudiantes está en escuelas segregadas desaventajadas, 22\% en escuelas mixtas y $43 \%$ en escuelas segregadas aventajadas.

Cuadro 3: Dependencia administrativa de los establecimientos segregados y mezclados (Porcentajes de estudiantes y error standard)

\begin{tabular}{l|c|c|c|c|c|c}
\hline & \multicolumn{2}{|c|}{$\begin{array}{c}\text { Segregados } \\
\text { desaventajados }\end{array}$} & \multicolumn{2}{c|}{ Mezclados } & \multicolumn{2}{c}{$\begin{array}{c}\text { Segregados } \\
\text { aventajados }\end{array}$} \\
\hline Dependencia & Porcentaje & Error st & Porcentaje2 & Error st2 & Porcentaje & Error st3 \\
\hline Municipal & 55,4 & 3,58 & 42,8 & 8 & 6 & 3,34 \\
\hline P. Subvencionado & 44,6 & 3,58 & 57,1 & 8 & 69,5 & 3,42 \\
\hline P. Privado & - & - & 0,05 & 0,03 & 24,4 & 2,02 \\
\hline Total & 100 & & 100 & & 100 & \\
\hline
\end{tabular}

Fuente: bases de datos PISA 2012, elaboración propia.

\subsubsection{Tamaño de la localidad}

El Cuadro 4 muestra la distribución de los estudiantes de los tres grupos en establecimientos emplazados en localidades de distinto tamaño. En los pueblos pequeños y grandes ${ }^{8}$, la presencia de estudiantes segregados desaventajados es abrumadora en comparación

8 Ejemplos de pueblos chicos: Panimávida, Cumpeo, San Fabián de Alico, Niebla, Neltume, Cochrane. Ejemplos de pueblos grandes: Freirina, Punitaqui, El Quisco, Chanco, Tirúa, Lonquimay, Dalcahue. 
con los otros dos grupos (dados los tamaños de estos tres grupos). Llama la atención que los establecimientos segregados desaventajados concentren un porcentaje minoritario de sus estudiantes en la gran ciudad; en cambio, la ciudad pequeña y la ciudad intermedia ${ }^{9}$ son el hábitat más frecuente de estos estudiantes. Los estudiantes de escuelas segregadas aventajadas sí abundan en la gran ciudad y en la intermedia, en iguales proporciones, y escasean en los pueblos. El caso de los estudiantes de escuelas mixtas es parecido al anterior, pero con una presencia notoriamente predominante en las ciudades intermedias. Lamentablemente la base de datos no registra la ciudad o comuna de emplazamiento de las escuelas.

Cuadro 4: Tamaño de la localidad donde se ubican los establecimientos segregados y mezclados (Porcentajes de estudiantes y error standard)

\begin{tabular}{l|c|c|c|c|c|c}
\hline & \multicolumn{2}{|c|}{$\begin{array}{c}\text { Segregados } \\
\text { desaventajados }\end{array}$} & \multicolumn{2}{|c|}{ Mezclados } & \multicolumn{2}{|c}{$\begin{array}{c}\text { Segregados } \\
\text { aventajados }\end{array}$} \\
\hline Tamaño de la localidad & Porcentaje & Error st & Porcentaje & Error st2 & Porcentaje3 & Error st4 \\
\hline $\begin{array}{l}\text { Pueblo pequeño }(<3.000 \\
\text { hab.) }\end{array}$ & 7,3 & 2,79 & - & - & 0,7 & 0,42 \\
\hline $\begin{array}{l}\text { Pueblo grande (3.000 a } \\
\text { 15.000 hab.) }\end{array}$ & 22,6 & 3,57 & 5,3 & 3,01 & 3,1 & 2,65 \\
\hline $\begin{array}{l}\text { Ciudad pequeña (15.000 } \\
\text { a 100.000 hab.) }\end{array}$ & 31,1 & 5 & 19,2 & 6,63 & 25,8 & 5,46 \\
\hline $\begin{array}{l}\text { Ciudad intermedia } \\
\text { (100.000 a 1.000.000 } \\
\text { hab.) }\end{array}$ & 30,4 & 5,2 & 52,8 & 9,08 & 35 & 5,72 \\
\hline $\begin{array}{l}\text { Gran ciudad ( } \rightarrow \\
\text { 1.000.000 hab.) }\end{array}$ & 8,7 & 3,06 & 22,7 & 7,44 & 35,5 & 5,14 \\
\hline \begin{tabular}{l} 
Total \\
\hline
\end{tabular} & 100 & & 100 & & 100 & \\
\hline
\end{tabular}

Fuente: bases de datos Pisa 2012, elaboración propia.

\subsubsection{Selección académica}

El siguiente criterio de descripción se refiere al grado en que los establecimientos de los tres grupos de la muestra realizan selección académica de sus estudiantes. En el Cuadro 5 puede apreciarse lo extendidas que están las prácticas de selección académica en los tres

9 Ejemplos de ciudades pequeñas: Vallenar, San Felipe, Cauquenes, La Unión, Castro. De ciudades intermedias: Iquique, Antofagasta, Talca, Los Ángeles, Temuco, Puerto Montt. 
grupos, pues la mayoría lo hace en mayor o menor medida, toda vez que la prohibición legal de esta práctica no rige en el nivel escolar de los estudiantes de la muestra. Los establecimientos segregados desaventajados son los que menos seleccionan a sus estudiantes por razones académicas; un tercio de sus estudiantes asiste a escuelas cuyo director declara inexistencia de esta práctica; superior al $16 \%$ de los estudiantes de escuelas mixtas cuyo director dice lo mismo, y muy superior al 3\% de sus pares de escuelas segregadas aventajadas. Estas últimas escuelas son las que más seleccionan: el $66 \%$ de sus estudiantes asiste a establecimientos donde el director reconoce que siempre aplica al menos uno de los dos factores de selección académica. Los establecimientos con mixtura social están en una situación intermedia: seleccionan menos que los segregados aventajados y más que los segregados desaventajados.

Cuadro 5: Selección académica en los establecimientos segregados y mezclados (Porcentajes de estudiantes y error standard)

\begin{tabular}{l|c|c|c|c|c|c}
\hline & \multicolumn{2}{|c|}{$\begin{array}{c}\text { Segregados } \\
\text { desaventajados }\end{array}$} & \multicolumn{2}{c|}{ Mezclados } & \multicolumn{2}{c}{$\begin{array}{c}\text { Segregados } \\
\text { aventajados }\end{array}$} \\
\cline { 2 - 7 } & Porcentaje & Error st & Porcentaje2 & Error st3 & Porcentaje4 & Error st5 \\
\hline $\begin{array}{l}\text { Ambos factores* nunca } \\
\text { son considerados }\end{array}$ & 33,7 & 4,7 & 15,8 & 5,15 & 2,9 & 2,12 \\
\hline $\begin{array}{l}\text { Al menos un factor es } \\
\text { considerado a veces, } \\
\text { pero nunca siempre }\end{array}$ & 45,8 & 6,4 & 54,8 & 9,02 & 31 & 6,58 \\
\hline $\begin{array}{l}\text { Al menos un factor es } \\
\text { siempre considerado }\end{array}$ & 20,5 & 4,76 & 29,4 & 8,53 & 66,1 & 6,41 \\
\hline Total & 100 & & 100 & & 100 & \\
\hline
\end{tabular}

*Nota: Los factores son "selección académica al ingreso" y "rendimiento académico como causal de abandono del establecimiento". Fuente: bases de datos PISA 2012, elaboración propia.

\subsection{Composición social y rendimiento en matemática}

Los datos del Cuadro 6 detallan la relación entre la composición social del alumnado en las escuelas, y su rendimiento en matemática, en términos del déficit o superávit de puntos que obtienen en la prueba, respecto del puntaje predicho para ellos según el NSE de sus familias. Conviene advertir del siguiente cambio en los tres grupos originales de composición social. Dado el hallazgo mencionado algunas páginas 
más arriba en relación a la composición interna del grupo de escuelas segregadas aventajadas, se consideró de interés dividir este grupo en dos, separando a las escuelas particulares privadas en un cuarto grupo (las más segregadas de todas). De este modo, el grupo de segregadas aventajadas consigue algo más de mixtura social, aunque en menor medida que las escuelas mixtas.

Cuadro 6: Residuos del logro en matemática de los estudiantes de los cinco quintiles de NSE, para los cuatro grupos de escuelas (Media de puntos de la escala de logros)

\begin{tabular}{l|c|c|c|c|c|c|c|c}
\hline & \multicolumn{2}{|c|}{$\begin{array}{c}\text { Segregadas } \\
\text { desaventajadas }\end{array}$} & \multicolumn{2}{c|}{ Mixtas } & \multicolumn{2}{c|}{$\begin{array}{c}\text { Segregadas } \\
\text { aventajadas }\end{array}$} & \multicolumn{2}{|c}{ Particulares privadas } \\
\hline NSE & $\begin{array}{c}\text { Media } \\
\text { residuos }\end{array}$ & Error st & $\begin{array}{c}\text { Media } \\
\text { residuos2 }\end{array}$ & Error st2 2 & $\begin{array}{c}\text { Media } \\
\text { residuos4 }\end{array}$ & Error st5 & $\begin{array}{c}\text { Media } \\
\text { residuos6 }\end{array}$ & Error st7 \\
\hline Q1 & 0,7 & 3,42 & $10,7 *[1]$ & 9,96 & 56,9 & 15,66 & - & - \\
\hline Q2 & $-8,4$ & 3,57 & $3,4^{*}[2]$ & 8,43 & 40,3 & 9,4 & $26,9 *[3]$ & 48,39 \\
\hline Q3 & $-17,7$ & 4,53 & $-8,0 *[4]$ & 6,54 & 33,2 & 6,8 & $61,7 *[5]$ & 14,71 \\
\hline Q4 & -33 & 6,61 & $-30,3^{*}[6]$ & 7,37 & 14,5 & 6,27 & 36,8 & 8,38 \\
\hline Q5 & $-55,7$ & 9,4 & $-56,2^{*}[7]$ & 9,97 & 3,6 & 7,84 & 35,2 & 3,22 \\
\hline
\end{tabular}

Fuente: bases de datos PISA 2012, elaboración propia.

El análisis residual de la regresión del NSE de los estudiantes sobre sus puntajes en la prueba de matemática muestra que aquellos que asisten a establecimientos segregados desaventajados, rinden menos de lo que se esperaría de ellos dados sus antecedentes socioculturales familiares; mientras más alto es su background sociocultural, más grande es la pérdida. En los establecimientos donde predomina la mixtura social, los estudiantes de los dos primeros quintiles obtienen casi 11 y 3 puntos más, respectivamente, de lo que se esperaría de ellos dado su background familiar; en cambio, los estudiantes de los tres quintiles restantes obtienen 8, 30 y 57 puntos menos, respectivamente, de lo que se esperaría de ellos. Por otra parte, los estudiantes de todos los quintiles de NSE que asisten a establecimientos segregados aventajados, rinden más de lo que se esperaría de ellos dados sus antecedentes socioculturales familiares; nótese la magnitud de las ganancias que obtienen los estudiantes del primer y segundo quintil: 57 y 40 puntos más respectivamente. Algo similar a lo anterior ocurre en los establecimientos particulares 
pagados, donde ganan los quintiles 3, 4, y 5 (especialmente el quintil 3); el quintil 2 aparece con ganancia, pero el número de casos es muy reducido.

Las pruebas de significación estadística permiten aquilatar mejor estos resultados. Se computaron las diferencias de las medias de los residuos de los estudiantes de un mismo quintil, entre los cuatro grupos de composición social y el error estándar de esas diferencias ${ }^{10}$. Así, la ventaja que obtiene un estudiante de cualquiera de los quintiles de asistir a una escuela mixta en vez de asistir a una escuela segregada desaventajada, no alcanza significación estadística; las magnitudes de las diferencias entre los residuos no son suficientemente grandes y/o los errores estándar son elevados. En cambio, la ventaja que obtiene un estudiante de cualquiera de los quintiles de asistir a una escuela segregada aventajada, en vez de asistir a una escuela desaventajada o una mixta, es significativa. Además, la ventaja que obtiene un estudiante de asistir a una escuela particular privada en vez de asistir a una segregada aventajada es significativa solo para los quintiles 4 y 5 .

\subsection{Composición social y calidad de la enseñanza de matemática en los establecimientos}

Los grupos anteriores fueron comparados en cuanto a las oportunidades de aprender que proporcionan a los estudiantes y a la calificación profesional formal del plantel docente. En el Cuadro 7 , las medias de los índices revelan que, en general, la cualidad positiva de los atributos medidos en estas variables la posee en mayor medida el grupo de particulares privadas, seguido de los establecimientos segregados aventajados y luego el grupo de escuelas mixtas; las escuelas segregadas desaventajadas poseen los índices más bajos en todos los casos. Hay algunas excepciones, pero el patrón es claro. Nótese que los tres índices relacionados con oportunidades de aprendizaje en este último grupo son negativos; también son negativos en las escuelas mixtas en dos de estos índices, lo que estaría indicando

10 La diferencia entre las medias de dos grupos es significativa si esa diferencia dividida por su error estándar, da un cuociente superior al intervalo +-1,96. 
que estas escuelas entregan oportunidades de aprendizaje más bien precarias en comparación con la media internacional.

Cuadro 7: Indicadores de calidad de la enseñanza de matemática en los establecimientos segregados y mixtos (Medias de los índices)

\begin{tabular}{|c|c|c|c|c|c|c|c|c|}
\hline \multirow[b]{2}{*}{ Variables de la escuela } & \multicolumn{2}{|c|}{$\begin{array}{c}\text { Segregados } \\
\text { desaventajadas }\end{array}$} & \multicolumn{2}{|c|}{ Mixtos } & \multicolumn{2}{|c|}{$\begin{array}{l}\text { Segregados } \\
\text { aventajadas }\end{array}$} & \multicolumn{2}{|c|}{$\begin{array}{c}\text { Particulares } \\
\text { privados }\end{array}$} \\
\hline & Media & Error st & Media 2 & Error st 3 & Media 4 & Error st 5 & Media 6 & Error st7 \\
\hline $\begin{array}{l}\text { Oportunidad } \\
\text { de aprendizaje } \\
\text { (matemática aplicada) }\end{array}$ & $-0,11$ & 0,03 & 0,04 & 0,06 & 0,01 & 0,04 & 0,12 & 0,04 \\
\hline $\begin{array}{l}\text { Oportunidad } \\
\text { de aprendizaje } \\
\text { (matemática pura) }\end{array}$ & $-0,33$ & 0,03 & $-0,11$ & 0,04 & 0,15 & 0,04 & 0,29 & 0,03 \\
\hline $\begin{array}{l}\text { Oportunidad } \\
\text { de aprendizaje } \\
\text { (conceptos } \\
\text { matemáticos) }\end{array}$ & $-0,19$ & 0,03 & $-0,01$ & 0,05 & 0,36 & 0,05 & 0,83 & 0,04 \\
\hline Docentes titulados & 0,17 & 0,03 & 0,23 & 0,07 & 0,16 & 0,05 & 0,42 & 0,07 \\
\hline $\begin{array}{l}\text { Docentes con } \\
\text { licenciatura }\end{array}$ & 0,88 & 0,02 & 0,96 & 0,02 & 0,94 & 0,03 & 0,98 & 0,01 \\
\hline $\begin{array}{l}\text { Docentes de } \\
\text { matemática con } \\
\text { licenciatura }\end{array}$ & 0,51 & 0,05 & 0,56 & 0,07 & 0,62 & 0,06 & 0,61 & 0,06 \\
\hline
\end{tabular}

Fuente: bases de datos PISA 2012, elaboración propia.

No obstante, no todas las diferencias encontradas son estadísticamente significativas. Se computaron las diferencias entre las medias de los tres grupos para todas las variables del cuadro anterior y el error estándar de esas diferencias, para determinar su significación estadística. El Cuadro 8 muestra las comparaciones entre grupos de escuelas que resultaron significativas (en negritas, última columna). 
116 ESCUELAS INCLUSIVAS EN EL CONTEXTO DE SEGREGACIÓN SOCIAL DEL SISTEMA ESCOLAR CHILENO - I. Ortiz

Cuadro 8: Diferencias significativas y no significativas entre las medias de los indices de calidad de la enseñanza de los cuatro grupos de escuelas

\begin{tabular}{|c|c|c|c|c|}
\hline Variables & Comparación* & $\begin{array}{l}\text { Diferencia de } \\
\text { Medias }\end{array}$ & Error st & Diferencia/error st \\
\hline \multirow{6}{*}{$\begin{array}{l}\text { Oportunidades } \\
\text { de aprendizaje } \\
\text { (matemática } \\
\text { aplicada) }\end{array}$} & grupo1_2 & $-0,15$ & 0,07 & $-2,28$ \\
\hline & grupo1_3 & $-0,122$ & 0,05 & $-2,41$ \\
\hline & grupo1_4 & $-0,234$ & 0,06 & $-4,23$ \\
\hline & grupo2_3 & 0,028 & 0,07 & 0,43 \\
\hline & grupo2_4 & $-0,084$ & 0,07 & $-1,15$ \\
\hline & grupo3_4 & $-0,113$ & 0,06 & $-1,99$ \\
\hline \multirow{6}{*}{$\begin{array}{l}\text { Oportunidades } \\
\text { de aprendizaje } \\
\text { (matemática pura) }\end{array}$} & grupo1_2 & $-0,218$ & 0,06 & $-3,51$ \\
\hline & grupo1_3 & $-0,473$ & 0,05 & $-8,64$ \\
\hline & grupo1_4 & $-0,617$ & 0,04 & $-14,1$ \\
\hline & grupo2_3 & $-0,255$ & 0,06 & $-4,35$ \\
\hline & grupo2_4 & $-0,398$ & 0,06 & $-7,07$ \\
\hline & grupo3_4 & $-0,143$ & 0,05 & $-2,7$ \\
\hline \multirow{6}{*}{$\begin{array}{l}\text { Oportunidades } \\
\text { de aprendizaje } \\
\text { (conceptos } \\
\text { matemáticos) }\end{array}$} & grupo1_2 & $-0,175$ & 0,06 & $-2,9$ \\
\hline & grupo1_3 & $-0,549$ & 0,06 & $-9,63$ \\
\hline & grupo1_4 & $-1,018$ & 0,05 & $-18,96$ \\
\hline & grupo2_3 & $-0,374$ & 0,07 & $-5,19$ \\
\hline & grupo2_4 & $-0,842$ & 0,07 & $-12,65$ \\
\hline & grupo3_4 & $-0,468$ & 0,07 & $-6,8$ \\
\hline \multirow{6}{*}{$\begin{array}{l}\text { Proporción de } \\
\text { docentes titulados }\end{array}$} & grupo1_2 & $-0,058$ & 0,08 & $-0,72$ \\
\hline & grupo1_3 & 0,016 & 0,06 & 0,26 \\
\hline & grupo1_4 & $-0,247$ & 0,08 & $-3,24$ \\
\hline & grupo2 3 & 0,074 & 0,09 & 0,83 \\
\hline & grupo2_4 & $-0,188$ & 0,11 & $-1,79$ \\
\hline & grupo3_4 & $-0,262$ & 0,09 & $-3,03$ \\
\hline \multirow{6}{*}{$\begin{array}{l}\text { Proporción de } \\
\text { docentes con } \\
\text { licenciatura }\end{array}$} & grupo1_2 & $-0,078$ & 0,03 & $-2,73$ \\
\hline & grupo1_3 & $-0,058$ & 0,04 & $-1,41$ \\
\hline & grupo1_4 & $-0,102$ & 0,03 & $-3,9$ \\
\hline & grupo2_3 & 0,02 & 0,04 & 0,55 \\
\hline & grupo2_4 & $-0,024$ & 0,02 & $-1,52$ \\
\hline & grupo3_4 & $-0,044$ & 0,03 & $-1,32$ \\
\hline \multirow{8}{*}{$\begin{array}{l}\text { Proporción de } \\
\text { docentes de } \\
\text { matemática con } \\
\text { licenciatura }\end{array}$} & grupo1_2 & $-0,044$ & 0,08 & $-0,54$ \\
\hline & grupo1_3 & $-0,104$ & 0,08 & $-1,34$ \\
\hline & grupo1_4 & $-0,094$ & 0,08 & $-1,23$ \\
\hline & grupo2_3 & $-0,06$ & 0,1 & $-0,62$ \\
\hline & grupo2_4 & $-0,05$ & 0,09 & $-0,56$ \\
\hline & grupo3_4 & 0,009 & 0,08 & 0,11 \\
\hline & grupo2_4 & $-0,185$ & 0,09 & $-1,96$ \\
\hline & grupo3_4 & $-0,183$ & 0,09 & $-2,12$ \\
\hline
\end{tabular}

Fuente: bases de datos PISA 2012, elaboración propia.* Grupo 1=segregadas desaventajadas; grupo 2=mixtas; grupo 3=segregadas desaventajadas: grupo 4=particulares privadas. 
Interesa destacar el comportamiento de los tres índices de oportunidades de aprendizaje (matemática aplicada, matemática pura y conceptos matemáticos): las diferencias entre los grupos en estudio resultaron significativas, validando así el patrón sugerido arriba. Con una salvedad: las oportunidades de aprender matemática aplicada no resultaron significativamente diferentes para el grupo mixto en comparación con los dos grupos más aventajados; sí en comparación con el grupo segregado desaventajado.

En cambio, las diferencias de dotación de docentes de matemática con licenciatura no son significativas para ninguno de los grupos; las diferencias de dotación de docentes titulados y de docentes con licenciatura son significativas solo para algunas comparaciones entre grupos.

\section{Discusión y conclusiones}

La mayoría de los estudiantes chilenos de 15 años estudia en establecimientos socialmente segregados, situación que había sido puesta en evidencia y analizada en diversos estudios anteriores. Menos reconocida es la existencia de establecimientos con mixtura social donde estudia el 22,8\% de los estudiantes de 15 años. El procedimiento aquí utilizado para estimar el nivel de inclusión social del sistema escolar permite visibilizar los establecimientos concretos que se encuentran en una u otra situación y observar su composición social interna. Esto tiene un valor informativo adicional a otros índices de inclusión/segregación social usados por PISA en los informes oficiales de resultados, como por ejemplo, el porcentaje de la variabilidad total del NSE de los alumnos que depende de la variabilidad encontrada entre escuelas y al interior de estas.

La magnitud de este grupo es indicativa del nivel de inclusión o exclusión social del sistema escolar. Es interesante consignar que los países participantes del estudio PISA presentan también diversos grados de segmentación social de sus establecimientos. Usando la misma metodología de clasificación aplicada en este estudio, OECD (2009) reporta que países de reconocida equidad social como Suecia y Finlandia, en la evaluación PISA 2003 tienen un 14\% y 19\% de 
estudiantes, respectivamente, segregados en establecimientos de bajo NSE, en tanto que el 19\% y el 15\% lo hace en establecimientos de alto NSE. En el otro extremo, países como Hungría llegan al 35\% y $36 \%$ en ambas condiciones.

Los resultados confirman un hallazgo consensual en la literatura, a saber, que la mixtura social de las escuelas es favorable para el rendimiento de los estudiantes de bajo NSE que asisten a ellas. Este hallazgo no alcanza significación estadística en este estudio al comparar las escuelas inclusivas con las segregadas desaventajadas, pero sí la tiene al comparar ambos grupos con las escuelas segregadas aventajadas, las cuales poseen un nivel menor de mixtura social. Este hallazgo sugiere que no cualquier composición mixta de las escuelas puede hacer una diferencia importante: los estudiantes desaventajados favorecerían sustantivamente su aprendizaje en contextos donde predominan los compañeros aventajados (no necesariamente particulares privados). Por otra parte, estos resultados se alinean con aquellos estudios que reportan una situación desfavorable para el rendimiento de los estudiantes de NSE superiores que asisten a establecimientos inclusivos.

Los estudiantes particulares privados asisten a escuelas segregadas aventajadas. Los del sector municipal asisten principalmente a escuelas segregadas desaventajadas; también asisten a establecimientos con mixtura social, aunque en menor medida. En cambio, los estudiantes particulares subvencionados se distribuyen en forma más homogénea entre los tres contextos de composición social. Se sabe que el sector particular subvencionado concentra más de la mitad de la matrícula de educación media, por lo que se transforma en el mayor reservorio de mixtura social en términos relativos y absolutos. Esto puede explicarse porque este sector recibe estudiantes de un espectro social más amplio que los establecimientos municipales y los particulares privados, más circunscritos estos a los NSE inferiores y superiores, respectivamente. Los establecimientos particulares subvencionados, en una proporción considerable, presentan una segregación inclinada a los NSE superiores, seguramente asociada a un copago alto. Pero son también el lugar de encuentro de estudiantes de familias con diversos antecedentes socioeconómicos. Es posible imaginar, por ejemplo, 
un colegio particular subvencionado con copago de $\$ 10.000$ pesos como lugar de encuentro de estudiantes de familias del quintil 1 y 2 muy esforzadas y con altas expectativas educacionales para sus hijos (quizás únicos), con estudiantes provenientes de familias del quintil 3 y 4 con varios hijos en el mismo colegio.

La selección académica, en mayor o menor grado, es una práctica extendida en la mayoría de los establecimientos, incluidos los que concentran estudiantes de NSE inferiores. Este hecho condiciona la lectura de los resultados de logro. Cierto, los estudiantes de los quintiles 1 y 2 que asisten a establecimientos con mixtura social obtienen un rendimiento en la prueba de matemática superior a lo estimado para ellos dado su background socioeconómico, superando así a sus pares de la misma condición social que estudian en establecimientos segregados desaventajados. Pero algunos de ellos son seleccionados académicamente. Estos resultados plantean la interrogante de si las escuelas inclusivas -y las segregadas aventajadasreproducirían los buenos resultados con estudiantes de NSE modesto, sin mediar la selección académica.

De acuerdo al reporte de los propios estudiantes, los establecimientos con mixtura social ofrecen más oportunidades de aprender matemática que los segregados desaventajados, en tanto que los particulares privados presentan las mayores oportunidades, seguidos de los segregados aventajados. La certificación formal de la dotación docente no parece tener una asociación clara con esas oportunidades, pero es claro que la calidad de la enseñanza varía entre esas escuelas. Este hecho pone límites a una interpretación según la cual sería el intercambio entre pares el responsable de los efectos composicionales encontrados. Si bien el diseño del estudio y la ausencia de variables de interacción entre pares no permiten descartar esta interpretación, ella tendría que competir con una explicación basada en la calidad diferencial de la enseñanza.

El hecho de que el estudio muestre diferencias de efectividad de la enseñanza asociadas con la composición social de las escuelas, sugiere que políticas antisegmentación del sistema escolar, si no van acompañadas de medidas de gestión interna de las escuelas destinadas 
a fortalecer la enseñanza, no tendrían impacto en los aprendizajes. Es cierto que el aumento de la efectividad escolar podría considerarse una externalidad positiva de las políticas antisegmentación; se podría pensar que los profesores y la gestión pedagógica acomodarán, tarde o temprano, la calidad de la oferta de enseñanza a las características del nuevo público escolar que tienen en frente. Pero en un contexto de aumento de escuelas inclusivas, por un lado, con escasez de buenos profesores y de buenas prácticas de gestión pedagógica en las escuelas, por otro, tales externalidades positivas podrían demorarse en arrancar.

Finalmente, la cantidad de estudiantes que asiste a establecimientos inclusivos es significativa, pese a ser minoritaria. Justifica un esfuerzo por conocerlos mejor, por ejemplo, averiguar los factores que explican su presencia en medio de un sistema predominantemente segregado. ¿Obedecen a una intencionalidad educativa, a un proyecto educativo inclusivo de ciertos sostenedores y comunidades?, ¿son una formación natural en comunas del país con heterogeneidad social? Por supuesto, interesa profundizar en el comportamiento del aprendizaje en estos contextos y qué resultados tienen en términos de convivencia y tolerancia sociales.

\section{Referencias}

Angrist, J. D. (en prensa). The perils of peer effects. Labour Economics. Recuperado de http://dx.doi.org/10.1016/j.labeco.2014.05.008

Carrell, S. E., Sacerdote, B. I., \& West, J. E. (2013). From natural variation to optimal policy? The importance of endogenous peer group formation. Econometrica, 81(3), 855-882.

Cerón, F. y Lara, M. (2011). Factores asociados con el rendimiento escolar: Simce 2010 Matemática $4^{\circ}$ básico e Inglés $3^{\circ}$ medio. Documento de Trabajo No 2, Sistema Nacional para la Medición de la Calidad de la Educación. Santiago: Ministerio de Educación.

Harris, D. N. (2010). How do school peers influence student educational outcomes? Theory and evidence from economics and other social sciences. Teachers College Record, 112(4), 1163-1197.

Hattie, J. (2002). Classroom composition and peer effects. International Journal of Educational Research, 37, 449-481. 
Manzi, J. (2007). Selección de estudiantes en el sistema escolar chileno: ¿La buena educación debe ser solo para los mejores? En J. J. Brunner y C. Peña (Eds.), La reforma del sistema escolar: aportes para el debate (pp. 203-220). Santiago: Universidad Diego Portales.

McEwan, P. (2003). Peer effects on student achievement: evidence from Chile. Economics of Education Review, 22, 131-141.

McDonnell, L. (1995). Opportunity to learn as a research concept and policy instrument. Educational Evaluation and Policy Analysis, 17(3), 305-319.

Mizala, A. \& Torche, F. (2012). Bringing the schools back in: the stratification of educational achievement in the Chilean voucher system. International Journal of Educational Development, 32, 132-144.

Mullis, I.V.S., Martin, M. O., Foy, P., \& Arora, A. (2012). TIMSS 2011 International results in Mathematics. Chestnut Hill, MA: TIMSS \& PIRLS International Study Center, Boston College.

Organisation for Economic Co-operation and Development (2009). PISA Data analysis manual, SPSS (2 ${ }^{\mathrm{a}}$ ed.). Paris: OECD Publishing.

Organisation for Economic Co-operation and Development (2010). PISA 2009 Results: overcoming social background: equity in learning opportunities and outcomes, Volume II. Paris: OECD Publishing.

Organisation for Economic Co-operation and Development (2013). PISA 2012 Results: excellence through equity: giving every student the chance to succeed, Volume II. Paris: OECD Publishing. http://dx.doi. org/10.1787/9789264201132-en

Parr, J. M., \& Townsend, M. A. (2002). Environments, processes, and mechanisms in peer learning. International Journal of Educational Research, 37, 403-423.

Perry, L. B., \& McConney, A. (2010). Does the SES of the school matter? An examination of socioeconomic status and student achievement using PISA 2003. Teachers College Record, 112(4), 1137-1162.

Peña, C. (2007). Por qué no debemos seleccionar. En J. J. Brunner y C. Peña (Eds.), La reforma del sistema escolar: aportes para el debate (pp. 245252). Santiago: Universidad Diego Portales.

Sapelli, C., \& Vial, B. (2005). Private v/s public voucher schools in Chile: new evidence on efficiency and peer effects. Documento de trabajo $\mathrm{N}^{\circ} 289$, Santiago: Instituto de Economía, P. Universidad Católica de Chile. Recuperado de www.economia.puc.cl/docs/dt_289.pdf 
Schmidt, W., \& McKnight, C. (1995). Surveying educational opportunity in mathematics and science: an international perspective. Educational Evaluation and Policy Analysis, 17(3), 337-353.

Schmidt, W., McKnight, C., Valverde, G., Houang, R., \& Wiley, D. (1997). Many visions, many aims. Volume 1. A cross national investigation of curricular intentions in school mathematics. Dordrecht: Kluwer Academic Publishers.

Schmidt, W., Zoido, P., \& Cogan, L. (2013). Schooling matters: opportunity to learn in PISA 2012. Education Working Papers, 95. OECD Publishing, Paris. http://dx.doi.org/10.1787/5k3v0hldmchl-en

Sousa, D. A., \& Tomlinson, C. A. (2011). Differentiation and the brain: how neuroscience supports the learner-friendly class. Bloomington, IN: Solution Tree Press.

Taut, S. y Escobar, J. (2012). El efecto de las características de los pares en el aprendizaje de estudiantes chilenos de enseñanza media. Informes Técnicos Mide UC. Santiago: Pontificia Universidad Católica de Chile.

Thrupp, M., Lauder, H., \& Robinson, T. (2002). School composition and peer effects. International Journal of Educational Research, 37(5), 483-504.

Tomlinson, C. A. (2005). Estrategias para trabajar con la diversidad en el aula. Buenos Aires: Paidós.

Valenzuela, J. P., Bellei, C. y De Los Ríos, D. (2009). Evolución de la segregación socioeconómica de los estudiantes chilenos y su relación con el financiamiento compartido. En Fondo de Investigación y Desarrollo en Educación (Ed.), Evidencias para políticas públicas en educación (pp. 231-284). Santiago de Chile: Ministerio de Educación.

Wilkinson, I. (2002). Introduction: peer influences on learning: where are they? International Journal of Educational Research, 37, 395-401.

Wilkinson, I., Parr, J., Fung, I., Hattie, J., \& Townsend, M. (2002). Discussion: modelling and maximizing peer effects in school. International Journal of Educational Research, 37, 521-535.

Willms, J.D. (2010). School composition and contextual effects on student outcomes. Teachers College Record, 112(4), 1008-1037.

Recibido: 05/01/2015

Aceptado: 23/04/2015 